\title{
Evolutionary approaches to sexually transmitted infections
}

\author{
Randolph M. Nesse ${ }^{1}$ and Betsy Foxman ${ }^{2}$ \\ ${ }^{1}$ Department of Psychiatry and ${ }^{2}$ Department of Epidemiology, University of Michigan, Ann Arbor, Michigan
}

Address for correspondence: Randolph M. Nesse, Department of Psychiatry, University of Michigan, East Hall, Room 3018, Ann Arbor, Ml 48109. nesse@umich.edu

Evolutionary approaches are particularly valuable for studies of sexually transmitted diseases. Methods for tracing evolutionary phylogenies have powerful new applications that use genetic data to trace the history of pathogens across millions of years, within outbreaks lasting years, and even within individuals. Equally valuable are less widely appreciated evolutionary methods for analyzing how host-pathogen co-evolution shapes extreme traits whose costs can be substantial. These and other applications of Darwinian medicine will improve understanding and treatment of sexually transmitted diseases.

Keywords: evolution; sexually transmitted disease; medicine

The vast majority of studies on sexually transmitted infections (STI) has focused on diagnosis, treatment (especially emerging antibiotic resistance), and pathogenesis. In recent years, evolutionary approaches to medicine and public health have suggested new questions with new answers about these and other aspects of STI. ${ }^{1-4}$ Two kinds of evolutionary explanations have proved particularly valuable. The first, explanations of phylogeny and origins, has been the focus of excellent work on sexually transmitted infections (STIs). The second, equally valuable, are analyses of how coevolution of pathogens and hosts explains specialized traits and patterns of virulence and trends in antibiotic resistance.

Phylogenetic studies have led to several general conclusions. First, sexually transmitted pathogens are nothing new; they have been interacting with hosts in nearly all species, including plants, for eons. ${ }^{5}$ Second, it is clear that organisms often make the transition from nongenital to genital transmission, and after this transition, new selection forces shape them and their hosts. The result is extraordinary pathogen specialization for sexual transmission and delicacy outside the protected genital niche.

In conjunction with the new availability of genetic data, phylogenetic methods have also made it possible to trace the origins of specific strains of pathogens in both long and short time frames, with more progress coming fast. The case of HIV is a dramatic exemplar, but many pathogens can now be traced with enormous specificity, enhancing outbreak investigations and providing insight into pathogen emergence and spread. In combination with evolutionary theory, molecular methods are proving crucial for epidemiology. ${ }^{6}$

The other less-recognized contribution of evolutionary biology is an analysis of the selection forces shaping sexually transmitted pathogens in cycle after cycle of host-parasite coevolution that makes them very special indeed. ${ }^{5,7,8}$ This is the realm of behavioral ecology, where every trait is analyzed in terms of trade-offs-the costs and benefits of variations. ${ }^{9}$ This perspective offers many opportunities for progress, albeit with methods less straightforward than those using genetic data to analyze phylogenies. It makes possible analysis of the adaptations of sexually transmitted organisms to their specific niche, with the attendant costs and benefits of those specializations. It also gives insight into how STIs can change behavior and even sex ratios.

For example, a sexually transmitted insect pathogen, Wolbachia, uses several strategies to increase the number of infected females in the population, including feminization, parthenogenesis, male killing, and sperm-egg incompatibility. ${ }^{10}$ Sperm from an infected male does not result in offspring unless the female is infected with the same strain of Wolbachia. ${ }^{11}$ Lest we believe humans are immune to such manipulation, some bacterial STIs (e.g., chlamydia and gonorrhea) cause infertility. 
Infertility has profound effects on population structure. ${ }^{12}$ It may also motivate seeking additional partners, thus spreading disease. Furthermore, in the not so distant past, a recommended cure for sexually transmitted diseases was to have sex with a virgin. ${ }^{13}$ Although we know that humans with STIs have a history of more sex partners, it is uncertain if STIs might increase mating frequency.

Organisms transmitted by sexual contact are shaped by innumerable cycles of host-pathogen coevolution. They develop specialized mechanisms for invading genital mucosa, but become delicate outside of the genital niche. The density of the host population is relatively less important for transmission than frequency of sexual contact. ${ }^{5}$ When frequency of mating is low, this selects for low virulence, causing chronic disease, although increased rates of contact may shape higher virulence. ${ }^{5,14-16}$

Scientists are increasingly taking the pathogen's point of view when analyzing interactions with hosts. This change in perspective brings needed attention to the complexities of coevolution and the profound impact of changes in the ecosystem. This is particularly relevant for STIs now, as our immune systems have been substantially modified from those of our ancestors as a result of changes in diet and antibiotics that change our microbiomes. ${ }^{17}$ Changes in exposure to helminths and other pathogens also change our immune systems so that inflammatory responses to certain stimuli are orders of magnitude higher than humans experienced prior to modernization. An evolutionary approach to trade-offs in immune responses is also developing quickly, with recognition that the substantial costs of inflammation exist because in the ancestral environment they give, on average, a net benefit in the long run. In the modern environment, the optimal amount of inflammation may be quite different, an opportunity for study with practical implications. ${ }^{18}$

Research into selection on behavior has begun, and yielded remarkably few behavioral adaptations to avoid STI acquisition. Primates in species especially subject to STIs do not inspect sexual partners more carefully, nor are they more choosy, although the effect of STIs on selection is confirmed by their higher white blood cell counts. ${ }^{19}$ The absence of behavioral adaptations to avoid infection is surprising and requires further investigation. One possible explanation is that dangerous partners are not readily identified, perhaps because pathogen strains are shaped to be covert; gonorrhea and chlamydia are frequently asymptomatic or cause only minor symptoms. It is also possible that the reproductive benefits of sex now are usually greater than the possible future costs of contracting a STI. However, $20-30 \%$ of women cannot conceive because of damage from STI in some areas where infections are prevalent. This would seem to create an enormous selection force that should have major effects on behavioral tendencies as well as immune function. Looking for such effects offers a fine opportunity for research.

Many scientists are using evolutionary ideas to understand how selection changes organisms. However, selection far more often keeps things the same. Every trait has a distribution, and individuals at the extremes usually have reproductive success lower than those in the middle. This applies to the intensity of the inflammatory response, the allocation of resources to immune defenses versus other investments, and it applies to sexual behavior tendencies. Looking carefully at the reproductive success of individuals at the extremes of relevant traits offers a substantial opportunity. This applies, of course, to pathogens as well as hosts. Because the generation time for most pathogens is much shorter than the human lifespan (e.g., 30 min for Escherichia coli), a five-day course of antibiotics may allow only resistant pathogens to survive, and some of those may have evolved ways to minimize the fitness costs of resistance mechanisms. Modern laboratory techniques can distinguish between de novo resistant mechanisms that arise following therapy from selection for an existing mutation or following the spread of a resistance mutation via recombination or horizontal gene transfer; however, interpreting the results and predicting emergence and spread requires theory. Evolutionary theory offers our best hope of using results of these analyses to keep a distance between ourselves and faster evolving pathogens. ${ }^{20}$

Evolutionary medicine emphasizes the role of changing environments and fast-evolving pathogens as two of six factors that need consideration when trying to explain vulnerability to disease; other factors include constraints on selection, tradeoffs, selection for reproduction at the cost of health, and the costs of defenses such as inflammation. ${ }^{2}$ All six factors could be approached systematically in research on STIs.

Research on the effects of changes in the microbiota, and changes in immune responses as a result of deficient early exposure to immune stimulation, 
is underway. For example, we already know that the presence of bacterial vaginosis, a condition characterized by a disrupted microbial community, is associated with an increased risk of acquiring and transmitting HIV. Genital herpes infection also increases rates of HIV acquisition and transmission. ${ }^{21}$ Further research is urgently needed, because changing sexual behaviors are changing transmission dynamics in ways that will shape certain pathogens for faster transmission. This is particularly the case for transmission of herpes viruses in response to recent increases in oral-genital sex.

The effects of vaccines as a force of selection are also on the radar, but they could be addressed more explicitly. Research showing that vaccination for Marek's disease in chickens resulted in increased virulence of this viral pathogen suggests the need for systematic research. ${ }^{22}$ This is being considered by epidemiologists who are watching changes in human papilloma virus because of vaccination. It would be tragic if we did not take full advantage of our knowledge of how vaccination can be a selection force to try to anticipate and avoid public health problems.

Curiously, one of the foundations of evolutionary understanding of sex remains uncertain. ${ }^{23-25}$ Sexual reproduction passes on only half as many of a female's genes as nonsexual reproduction. You would think that asexual clades would soon displace sexual ones. That is exactly what happens in lizards that evolve through asexual reproduction. Sex persists, however, in large part because of selection pressures from infectious disease. In the field, this is observed in population crashes in asexual clades, which, being genetically identical, are prone to being wiped out by a pathogen. Thus, sex protects us against infection, even as it makes us vulnerable. We anticipate much will come from the synthesis of evolution, epidemiology, and molecular methods applied to the study of sexually transmitted infections.

\section{Conflicts of interest}

The authors declare no conflicts of interest.

\section{References}

1. Nesse, R.M. \& S.C. Stearns. 2008. The great opportunity: evolutionary applications to medicine and public health. Evol. Appl. 1: 28-48.

2. Nesse, R.M. \& G.C. Williams. 1994. Why We Get Sick - The New Science of Darwinian Medicine. Times Books. New York, NY.
3. Stearns, S.C. \& J.C. Koella. 2008. Evolution in Health and Disease. Oxford University Press. Oxford, New York.

4. Gluckman, P., A. Beedle \& M. Hanson. 2009. Principles of Evolutionary Medicine. Oxford University Press. Oxford, UK.

5. Lockhart, A.B., P.H. Thrall \& J. Antonovics. 1996. Sexually transmitted diseases in animals: ecological and evolutionary implications. Biol. Rev. Camb. Philos. Soc. 71: 415-471.

6. Foxman, B. 2011. Molecular Tools and Infectious Disease Epidemiology. Academic Press. New York.

7. Ewald, P. 1994. Evolution of Infectious Disease. Oxford University Press. New York.

8. Schmid-Hempel, P. \& D. Ebert. 2003. On the evolutionary ecology of specific immune defence. Trends Ecol. Evol. 18: 27-32.

9. Westneat, D.F. \& C.W. Fox. 2010. Evolutionary Behavioral Ecology. Oxford University Press. Oxford, New York.

10. Saridaki, A. \& K. Bourtzis. Wolbachia: more than just a bug in insects genitals. Curr. Opin. Microbiol. 13: 67-72.

11. Werren, J.H., L. Baldo \& M.E. Clark. 2008. Wolbachia: master manipulators of invertebrate biology. Nat. Rev. Microbiol. 6: 741-751.

12. Antonovics, J. 2009. The effect of sterilizing diseases on host abundance and distribution along environmental gradients. Proc. R. Soc. B Biol. Sci. 276: 1443-1448.

13. Lema, V. 1997. Sexual abuse of minors: emerging medical and social problem in Malawi. East Afr. Med. J. 74: 743-746.

14. Ewald, P.W. 1983. Host-parasite relations, vectors, and the evolution of disease severity. Annu. Rev. Ecol. Syst. 14: 465485.

15. Levin, B.R. 1996. The evolution and maintenance of virulence in microparasites. J. Short Form Workform 2: 93-102.

16. May, R.M. \& R.M. Anderson. 1983. Epidemiology and genetics in the coevolution of parasites and hosts. Proc. R. Soc. London, Ser. B. Biol. Sci. 219: 281-313.

17. Pallen, M.J. 2011. The human microbiome and hostpathogen interactions. In Metagenomics of the Human Body. K.E. Nelson, Ed.: 43-61. Springer. New York.

18. Schmid-Hempel, P. 2011. Evolutionary Parasitology: The Integrated Study of Infections, Immunology, Ecology, and Genetics. Oxford University Press. USA.

19. Nunn, C.L. 2003. Behavioural defences against sexually transmitted diseases in primates. Anim. Behav. 66: 37-48.

20. Read, A.F. \& S. Huijben. 2009. PERSPECTIVE: evolutionary biology and the avoidance of antimicrobial resistance. Evol. Appl. 2: 40-51.

21. Abu-Raddad, L.J. et al. 2008. Genital herpes has played a more important role than any other sexually transmitted infection in driving HIV prevalence in Africa. PLoS ONE 3: e2230.

22. Davison, F. \& V. Nair. 2005. Use of Mareks disease vaccines: could they be driving the virus to increasing virulence? Expert Rev. Vaccines 4: 77-88.

23. Williams, G.C. 1975. Sex and Evolution. Princeton University Press. Princeton, NJ.

24. Zuk, M. 2007. Riddled With Life : Friendly Worms, Ladybug Sex, and the Parasites that Make Us Who We Are. Harcourt. Orlando.

25. Hurst, L.D. \& J.R. Peck. 1996. Recent advances in understanding of the evolution and maintenance of sex. Trends Ecol. Evol. 11: 46-52. 